$\xi=2$ 줄

\title{
Comparative evaluation of anti-bacterial efficacy of silver nanoparticle, nanoparticle calcium hydroxide and calcium hydroxide against enterococcus faecalis biofilm- An In vitro study
}

\author{
Dr. V. Abirami ${ }^{1}$ *, Dr. A. Shafie Ahamed ${ }^{2}$, Dr. Bhavani ${ }^{3}$, Dr. Rajaraman $^{3}$ \\ ${ }^{1}$ Former postgraduate student, Dept. of conservative dentistry\& Endodontics, RMDCH, Annamalai University, Chidambaram \\ ${ }^{2}$ HOD, Dept. of conservative dentistry \&Endodontics, RMDCH, Annamalai University, Chidambaram \\ ${ }^{3}$ Senior lecturers, Dept. of conservative dentistry \&Endodontics, RMDCH, Annamalai University, Chidambaram \\ ${ }^{*}$ Corresponding author E-mail:
}

\begin{abstract}
The purpose of this study was to evaluate the antibacterial efficacy of silver Nanoparticles, Nanoparticle calcium hydroxide and calcium hydroxide as intracanal medicament against Enterococcus faecalis bio film formed on root dentin

The Minimal inhibitory concentration test and Anti microbial test were conducted for silver nanoparticle, Nanoparticle calcium hydroxide. Teeth were inoculated with E.faecalis strains for 1month to establish a standard mono species bio film model. Biofilm formation is confirmed using SEM. The intracanal medicaments were placed inside the canal and colony forming units (CFU) were counted before and 7 days later the placement of the medicament.

MIC for AGNP is $25 \mu \mathrm{g} / 200 \mu \mathrm{l}$ and for $\mathrm{NCH}$ is $12.5 \mu \mathrm{g} / 200 \mu \mathrm{l}$. The Antimicrobial test conducted shows zone of inhibition about $11 \mathrm{~mm}$

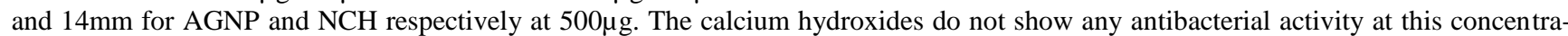
tion. In antibacterial efficacy test using CFU count, the AGNP and $\mathrm{NCH}$ shows $75 \%$ and $61 \%$ of antibacterial efficacy respectively whereas calcium hydroxide has exhibited just $11 \%$ of antibacterial efficacy. Silver Nanoparticles and nanoparticle calcium hydroxide had statistically significant difference in the reduction in the number of colonies when compared to calcium hydroxide by post hoc turkey test and p-value of $<0.01$ was considered as significant in this study.
\end{abstract}

Keywords: Silver Nanoparticles; Calcium Hydroxide; Intracranial Medicaments; E.Faecalis

\section{Introduction}

The basis of endodontic treatment depends on identifying and eliminating the causative factors in the development of apical periodontitis so that optimal healing can be achieved. The role of bacteria and their byproducts in the pathogenesis of apical periodontitis has been clearly established. An improved prognosis has been shown for the teeth after a negative culture has been obtained compared with teeth having a positive culture at the time of obturation. Therefore, the prime objective of treatment is to eliminate bacteria and sources of nutrient supply from root canal system. [1] It was well known that after the Instrumentation of the infected pulp space, some type of further disinfection would be required to enhance the chances for treatment success. The proven step to take is to apply an effective antimicrobial agent in the root canal for a predetermined time period to further eradicate the remaining bacteria. Enterococcus faecalis is the predominant micro-organism and occasionally the only species detected in root canals of teeth associated with persistent periradicular lesions. It is hardy microbe that possesses certain virulence factors including lytic enzymes, cytolysin, aggregation substances, pheromones, and lipoteichoic acid. E.faecalis is able to invade dentinal tubules and remain viable within the tubules for prolonged period of time, adhere and form bioflim on dentin under different environmental conditions, resist intracanal disinfectants, survive harsh conditions within root filled teeth. Historically, efforts to eliminate E.faecalis and its concomitant bioflim have been somewhat limited while using commonly used root canal disinfectants. [2].

Since its Introduction as an intracanal medicament in 1920 (HERMANN 1920) calcium hydroxide has been widely used in Endodontics. It is a strong alkaline substance, which has a $\mathrm{Ph}$ of approximately 12.5 . Various biological properties have been attributed to this substance, such as Antimicrobial activity (Bystrom et al 1985), tissue dissolving ability (Hasselgren et al 1988, Andersen et al 1992), because of such effects calcium hydroxide is the most commonly used Intracanal medication during root canal procedures. Its antibacterial property is generally related to the release of hydroxyl ions, which produces the lethal effects on bacterial cells including protein denaturation and damage to the bacterial cytoplasmic membranes and DNA. However, calcium hydroxide can be inactivated by dentin exudates from periapical area and microbial biomass. ${ }^{3}$ recently, much attention has been focused on Nanoparticle in many health care fields. With regard to their high surface-to-volume ratio, Nanoparticles are one of the most effective antibacterial agents. Silver nanoparticle (AgNPs) has been applied in many health care fields because of their broad spectrum bactericidal and virucidal properties. AgNPs have high surface- area-to-volume ratio 
and unique chemical and physical properties which results in increased reactivity. AgNPs shows multiple antibacterial mechanisms such as adherence and penetration into bacterial cel membrane and cell wall permeability. AGNPs with size in the range of 10- $100 \mathrm{~nm}$ showed powerful bactericidal potential against both gram positive and gram negative bacteria. [4] Similarly calcium hydroxide is a commonly used intra canal medicament but it lacks in ability of completely eliminating Enterococcus faecalis. Nanoparticle of calcium hydroxide $(\mathrm{NCH})$ would penetrate dentinal tubules deeper and at a greater concentration than calcium hydroxide. NCH have superior anti microbial activity against E.faecalis compared to conventional calcium hydroxide in culture media as well as dentin tubules.

So to evaluate the antibacterial efficacy of Nanoparticles, this study uses silver nanoparticle and Nanoparticle calcium hydroxide along with calcium hydroxide against mature E.faecalis bioflim.

\section{Materials and methods}

\subsection{Standardization of the specimens}

32 single rooted teeth (maxillary and mandibular anterior and mandibular premolars) were selected and stored in $0.5 \%$ Thymol. Teeth are sectioned $3 \mathrm{~mm}$ above CEJ and prepared with Protaper up to F3 maintaining patency. Smear layer removed (2 $\mathrm{ml}-6 \%$ NAOCL, $2 \mathrm{ml}-17 \%$ EDTA, $2 \mathrm{ml}-6 \%$ NAOCL with positive pressure irrigation using side vented needles). Teeth are wrapped in moist cloth and sterilized in autoclave. Teeth transferred to sterile vials containing $20 \mathrm{ml}$ sterile BHI (brain heart infusion) and kept in incubator at $37^{\circ} \mathrm{c}$ for $48 \mathrm{hrs}$ to check efficacy of sterilization procedures.

\subsection{Cultivation of enterococcus faecalis and specimen inoculation}

E.faecalis colonies are grown on BHI agar plates were suspended in $20 \mathrm{ml}$ sterile BHI broth for $8 \mathrm{hr}$. Five drops of BHI broth is added to $20 \mathrm{ml}$ broth for 4 hours. Bacterial suspension are adjusted to $1.5 \mathrm{X} 10{ }^{8} \mathrm{CFU} / \mathrm{ml}$ turbidity. 20 micro liters of bacterial inoculums added to vials containing the teeth suspended in sterile medium. Tubes closed and stored on $37^{\circ} \mathrm{c} / 30$ days to ensure adequate bacterial penetration in to dentinal tubules. Half of the inoculums broth for all specimens was replaced with $20 \mathrm{ml}$ of sterile BHI medium every 5 days. Bioflim formation is confirmed using SEM.

\subsection{Samples and microbiological analysis}

\subsubsection{Methodology for antibacterial effectiveness of intracanal medicament mixtures against E.faecalis}

silver nanoparticle, nanoparticle calcium hydroxide at different concentration of $500,250,125,50,12.5 \mu \mathrm{g} / 200 \mu \mathrm{l}$ is taken and grouped as Group1- silvernanoparticle,Group2-nanoaprticle calcium hydroxide ,Group3- calcium hydroxide, Group4 -

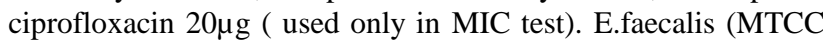
2729 ) is prepared according to 0.5 mac farland standard. Antibacterial activity and minimum inhibitory concentration is determined using disc diffusion method and micro broth dilution method respectively.

\section{Methodology for antibacterial activity of silver nanoparticle, nanoparticle calcium hydroxide, calcium hydroxide on mature biofilm E.faecalis}

\subsection{Preparation of bacterial samplings}

Teeth rinsed with sterile saline and wiped with alcohol outside .Sterile cotton pellets placed into chamber and access cavity is sealed with cavit.Apical foramen is sealed with hot glue to resemble closed system .Tooth is disinfected with $30 \%$

$\mathrm{H}_{2} \mathrm{O}_{2}$. Tooth surface coated with $10 \%$ tincture iodine and the surface is swabbed with $5 \%$ sodium thiosulphate.

\subsection{Initial sampling}

The Cavit and cotton pellets are removed and canal flushed with $2 \mathrm{ml}$ sterile saline and dried with sterile paper points. Sterile BHI broth inserted in to canals and removed with sterile paper points. This paper points are then placed into half of each BHI agar plates. Sterile BHI broth inserted and removed paper points are placed in $1 \mathrm{ml}$ test tube of BHI broth. The $1 \mathrm{ml}$ test tube is vortexes and aliquot of $0.1 \mathrm{ml}$ suspension was plated on to remaining half of BHI plates. The plates were incubated aerobically for 48 hours at $37^{\circ} \mathrm{c}$.

\subsection{Intracanal medicament placement}

Irrigation with $20 \mathrm{ml}$ of $1.5 \%$ NAOCL and $20 \mathrm{ml}$ of sterile saline with side vented needles and Canal dried .Silver nanoparticle powder ( average partial size $20-30 \mathrm{~nm}$, purity $-99 \%$, concentration 1000ppm) is mixed with distilled water to paste like consistency and placed inside the canal using lentulo spirals ( group $1-8$ teeth).Nano particle calcium hydroxide ( average particle size $30-50 \mathrm{~nm}$, purity $99.5 \%$ ) is mixed with distilled water to paste like consistency and placed inside the canal using lentulo spirals ( group $2-8$ teeth ).Full strength calcium hydroxide ( ultra cal XS) is placed inside the canal ( group 3- 8 teeth).Access cavity were closed with $4 \mathrm{~mm}$ Cavit and incubated at $37^{\circ} \mathrm{c}$ for 7 days. After 7 days canals are irrigated with $20 \mathrm{ml}$ of $17 \%$ EDTA with side vented needles and rinsed with saline and dried with paper points. Group $4-8$ teeth are negative controls, teeth with sterile incubations without medications.

\subsection{Final sampling}

Root canals of each tooth dried with sterile paper points and filled with reduced BHI broth. Three consecutive sterile paper points inserted in to the canal and paper points transferred to BHI agar plates. After collection of the first sample canals are filed with \# 10k files and instrumented for 10 seconds. Dentinal shavings from files transferred to test tube containing $1 \mathrm{ml}$ of BHI broth. The test tube containing dentinal shavings is vortexes for 10 seconds, aliquots of $0.1 \mathrm{ml}$ suspension were then placed on to another half of BHI agar plates and incubated aerobically at $37^{\circ} \mathrm{c}$ for 48 hours. CFU counted.

\section{Results}

All the materials are first subjected to antimicrobial test against E.faecalis by disc diffusion method. Ciprofloxacin $20 \mu \mathrm{g}$ is used as positive control for this test. In this test group I AGNPs showed $11 \mathrm{~mm}$ zone of inhibition at $500 \mu \mathrm{g}$ and Group II NCH showed $14 \mathrm{~mm}$ zone of inhibition at $500 \mu \mathrm{g}$. Group III CH do not exhibit any antimicrobial activity at this concentration. This clearly shows calcium hydroxide do not exhibit any antimicrobial property at very lower concentrations. The group IV positive

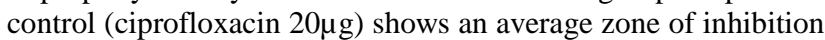
of $35 \mathrm{~mm}$ at $500 \mu \mathrm{g}$. Thereby this test clearly shows silver Nanoparticles and Nanoparticle calcium hydroxide exhibit antibacterial efficacy against E.faecalis at a concentration greater than or equal to $500 \mu \mathrm{g}$. Since calcium hydroxide did not exhibit any antimicrobial activity at lower concentration it is excluded in the test for Minimum Inhibitory Concentration. MIC test was performed for AGNPs and NCH using serial dilution method. The test results are shown as optical density values and percentage of 
inhibition. The test is repeated three times at every concentration in serial dilution and the average value is calculated for both the material. AGNPs shows an average optical density value of 1.082 and the percentage of inhibition is $37.77 \%$ at $25 \mu \mathrm{g} / 200 \mu \mathrm{l}$. $\mathrm{NCH}$ shows an average optical density value of 0.797 and percentage of inhibition is $54 \%$ at $12.5 \mu \mathrm{g} / 200 \mu \mathrm{l}$. The control group using ciprofloxacin showed optical density value as 1.738 . Thus MIC for AGNPs is $25 \mu \mathrm{g} / 200 \mu \mathrm{l}$ and $\mathrm{NCH}$ is $12.5 \mu \mathrm{g} / 200 \mu \mathrm{l}$. The reduction in CFU before and after the placement of intracanal medicament is depicted in the following bar diagram.

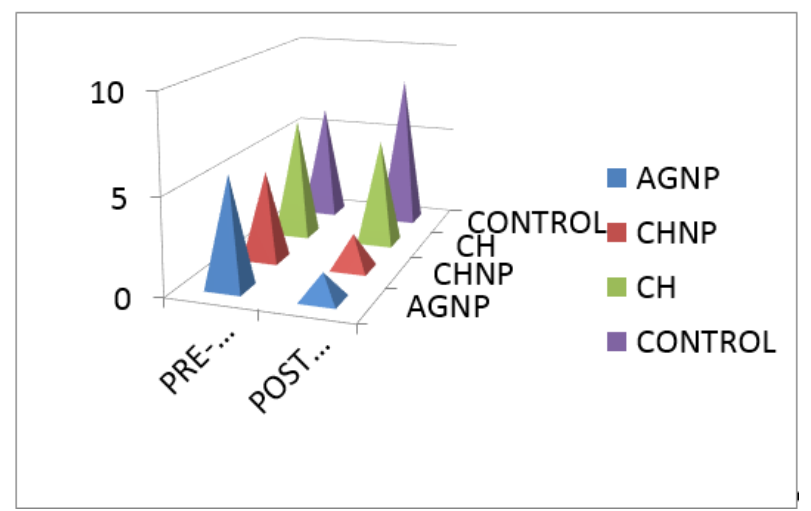

Fig. 1: Flow Chart Showing Reduction in CFU Before and after the Placement of Intracanal Medicaments among Groups.

The Mean CFU for group I before and after the placement of intracanal medicament is $5.74 \times 10^{2} / \mathrm{ml}$ and $1.37 \times 10^{2} / \mathrm{ml} \mathrm{respec-}$ tively and showed $76 \%$ reduction in culture formation. Similarly the mean CFU/ml for group II before and after 7 days of its placement are $4.67 \times 10^{2}$ and $1.81 \times 10^{2}$ respectively and showed $61 \%$ reduction in culture formation. Group III shows mean $\mathrm{CFU} / \mathrm{ml}$ before and after 7 days of its placement are $6.35 \times 10^{2}$ and $5.59 \times 10^{2}$ respectively and showed $11 \%$ reduction in culture formation. There is an increase in mean $\mathrm{CFU} / \mathrm{ml}$ in group IV which is the negative control group with no intracanal medicament placement. The culture test shows group I having highest Antibacterial efficacy among four groups by exhibiting $76 \%$ reduction in culture formation followed by group II $61 \%$ and group III $11 \%$.

Table 1: Showing Student T-Test among Groups

\begin{tabular}{ll} 
& Table 1: Showing Student T-Test among Groups \\
\hline Groups & T-Value \\
\hline Agnp & 14.3 \\
Nch & 8.34 \\
Ch & 1.54 \\
Control & 0.294 \\
\hline
\end{tabular}

\section{Discussion}

The method used in the present study simulates the clinical conditions in teeth with infected root canals, in which microorganisms may lodge in areas of difficult access for the root canal preparation and medicaments (Menezes et al.,2004) [5]. E.faecalis was chosen because of its resistance to calcium hydroxide based intracanal medicaments. (Heling et al.,1992, Basrani et al., 2003).this microorganism has been used in several studies( Evans et al.,2003, Siren et al.,2004, Delgado et al.,2010, Kandaswamy et al., 2010). [6-8]. The 21 day incubation period allowed the suspension of microorganism to diffuse throughout the root canal system (Heling et al 1992). The CFU is counted after 7 days of intracanal medicament placement to verify the presence of viable bacteria. This time frame is chosen based on the previous study by Menezes et al., 2004, Cardoso et al., 2008. According to sjogren et al., 1991, a minimum contact time of 7 days is necessary for calcium hydroxide to eliminate microorganism that may have survived cleaning and shaping procedures. [3]
The antimicrobial activity of calcium hydroxide depends on the amount of free $\mathrm{OH}^{-}$ions released and its contact time with microorganism in the root canal. The carriers used as vehicle for calcium hydroxide decreases its $\mathrm{PH}$ and affect its antimicrobial activity. Water must be present for the antimicrobial effect of calcium hydroxide (Safavi k et al., 2000). [9] Calcium hydroxide in water has a thixotrophic behavior and can be deposited in the canal with continuous agitation using lentulo spirals. Considering these facts, all the medicaments in this study are mixed to a paste like consistency and coated in the canal using lentulospirals. [1], [3].

The result of group I in this study correlates with the study done by $\mathrm{Wu}$ et al and kishen et al. [10] According to their results, syringe irrigation with $0.1 \%$ AGNPs solution did not damage the bioflim structure. On the other hand, bioflims treated with $0.02 \%$ AGNPs gel as a intracanal medicament for 7 days significantly eradicated the structure with least number of residual viable E.faecalis cells in comparison with $0.01 \%$ AGNPs gel and calcium hydroxide groups. The authors concluded that AGNPs should be applied as a medicament and not as an irrigant to exhibit potential activity against residual bacterial bioflims during root canal disinfection. [10] It is because the effectiveness of silver nanoparticle is directly proportional to the time it is in contact with bacterial cells. The probable mechanism of action of silver nanoparticle is the electrostatic attraction between the negative charge of the bacterial cell membrane and positively charged Nanoparticles (Kim et al., 2007). ). In terms of the molecular mechanisms of inhibitory action of silver ions on microorganisms, it has been shown that DNA loses its ability to replicate ( Feng et al.,2000) and the expression of ribosomal submit proteins and other cellular proteins and enzymes necessary for ATP production becomes inactivated (Yamanaka et al ., 2005). It has been hypothesized that silver ions affect membrane-bound respiratory enzymes (Bragg and Rainnie., 1974). The bacterial cells in contact with the silver Nanoparticles will take silver ions, which is possibly in turn will inhibit respiratory enzymes, and so help to facilitate reactive oxygen species/ free radical generation and subsequent damage to the cell membrane (Kim et al., 2007). [11] Similarly the results of group II in this study correlates with the study done by Omid Dianat et al [12] in which they concluded that the calcium hydroxide used in nanoparticle form as an intracanal medicament showed higher antibacterial efficacy than calcium hydroxide and the nanoparticle calcium hydroxide had better tubular penetration thereby producing better antibacterial efficacy.

Intergroup comparison is done between following groups: Group I and Group II, the culture reduction is insignificant $(\mathrm{p}>0.01)$. Similarly comparison in culture reduction between group III and group IV is also insignificant. All other combinations showed significant reduction in culture formation that is $\mathrm{CFU} / \mathrm{ml}(\mathrm{p}<$ 0.01 ).

Finally, Student- T - test is performed. Group I showed highest T value of 14.3, followed Group II 8.34, Group III 1.54 and Group IV showed the least value 0.294 .The group I exhibit highest antibacterial efficacy among four groups followed by group II,III,IV .

In our study GroupIII showed the least antibacterial efficacy. These results prove calcium hydroxide is inefficient in eliminating culture at a lower concentration in small time frame. In previous studies, it is reported that calcium hydroxide is a slow acting agent and should be kept in the canal for a longer period of time at a very high concentration.[14], [15] Most of the time it is difficult to achieve a high PH environment in root canal. It is mainly due to difficulty in depositing the intracanal medicament in the canal and poorer dentinal tubular penetration of the medicament. To overcome all these disadvantages, in this study calcium hydroxide has been used in Nanoparticle form (group II$\mathrm{NCH}$ ) and its antibacterial efficacy is compared with age known AGNPs (group I). And normal saline has been used as vehicle for Nanoparticles to avoid the disadvantages of vehicles interfering with its antibacterial efficacy due to its varied chemical 
composition. And all the intracanal medicament used in this study are applied inside the canal using lentulospirals considering the thixotropic behavior of the mixtures for better tubular penetration. [13]

This study clearly demonstrates that the increased antibacterial efficacy of Nanoparticle is mainly due to its smaller size than their bulk or original counterparts thereby increasing its contact surface area and better tubular penetration.

\section{Conclusion}

From the results of the study it could be concluded that Nanoparticles had significant antimicrobial activity against Enterococcus faecalis as evidenced by the reduction. This preliminary study suggests that both the new nanoparticle antimicrobial agents tested here have the potential to be used as intracanal medicaments. But concerns such as duration of the medicament placement, hypersensitivity reactions, toxicity and effect on other organisms should be addressed in the future studies.

\section{References}

[1] Amanda law. Harold messer. An Evidence- based analysis of the antibacterial effectiveness of intracanal medicaments. JOE 2004; vol 30; pg 689-694.

[2] M.Evans. J.K.Davies. G.Sundqvist. D.Figdor. Mechanism involved in the resistance of Enterococcus faecalis to calcium hydroxide. Int End J 2002; vol 35; pg 221-228. https://doi.org/10.1046/j.13652591.2002.00504.x.

[3] J.F.Siqueira, H.P.Lopes. Mechanism of antimicrobial activity of calcium hydroxide: a critical review. Int End j 1999; Vol 32; pg361-369.

[4] Jun sung kim et al. Antimicrobial effects of silver Nanoparticles.www.nanomedjournal.com [internet].2007; 95-101. Available from https://doi.org/10.1016/j.nano.2006.12.001.

[5] MENEZES M.M. M.C.VALERA. In vitro evaluation of the effectiveness of irrigants and intracanal medicaments on microorganisms within root canals. IEJ; VOL 37; ISSUE 5; Pg 311-319.

[6] J.F.Siqueira et al. Aetiology of root canal treatment failure: why well-treated teeth fail? Int End J.2001; vol34; pg1-10 https://doi.org/10.1046/j.1365-2591.2001.00396.x.

[7] Charles H.Stuart.Scott A.Schwartz. Enterococcus faecalis: its role in root canal treatment failure and current-concepts in retreatment.JOE.2006; vol32; pg93-98.

[8] D.Kandaswamy et al. Dentinal tubule disinfection with $2 \%$ Chlorhexidine gel, Propolis, morinda citrifolia juice $2 \%$ povidone iodine and calcium hydroxide. Int End J. May2010.vol43 pg419-423 https://doi.org/10.1111/j.1365-2591.2010.01696.x.

[9] Safavi KE, Spangberg LSW, Langeland K (1990) Root canal dentinal tubule disinfection. Journal of Endodontics 16, 207-10. https://doi.org/10.1016/S0099-2399(06)81670-5.

[10] Daming Wu.Anil kishen. Evaluation of the antibacterial efficacy of silver Nanoparticles against Enterococcus faecalis bioflim. JOE.Feb2014.vol40; pg285-290.

[11] R.P. Allaker. The use of Nanoparticles to control oral bioflim formation. J Dent Res.2010 89(11); 1175-1186 https://doi.org/10.1177/0022034510377794.

[12] Omid Dianat, Sara Saedi et al. Antimicrobial activity of Nanoparticle calcium hydroxide against enterococcus faecalis An in Vitro study. IEJ 2015; 10(1); 39-43.

[13] Teixeira FB, Levin LG, Trope M (2005). Investigation of PH at different dentinal sites after placement of calcium hydroxide dressing by two methods. Oral surgery, Oral medicine, Oral pathology, Oral radiology and endodontology 99, 511-6. https://doi.org/10.1016/j.tripleo.2004.07.023.

[14] Sjogren U, Figdor D, Spandber L, Sundqvist G. The anti-microbia effect of calcium hydroxide as short-term intracanal dressing. Int endod J 1991; 24119-125.

[15] Safavi KE, Dowden WE, Introcaso JH, Langeland K. A comparison of antimicrobial effects of calcium hydroxide and iodine-potassium iodide. J Endod 1985; 11; 454-456. https://doi.org/10.1016/S00992399(85)80086-8. 